\title{
Clinical Practice Patterns of Polycystic Ovary Syndrome Among Doctors of Different Specialties-An Indian Perspective
}

\author{
Deep Dutta, ${ }^{1}$ Saptarshi Bhattacharya, ${ }^{2}$ Deepak Khandelwal, ${ }^{3}$ Sameer Aggarwal, ${ }^{4}$ Rajiv Singla, ${ }^{5}$ Vineet Surana, ${ }^{6}$ Atul Dhingra, ${ }^{7}$ \\ and Sanjay Kalra ${ }^{8}$ \\ 1. Department of Endocrinology, Center for Endocrinology Diabetes Arthritis \& Rheumatism (CEDAR) Superspecialty Clinics, New Delhi, India; 2. Department of \\ Endocrinology, Max Superspecialty Hospital, Patparganj, New Delhi, India; 3. Department of Endocrinology, Maharaja Agrasen Hospital, Punjabi Bagh, New Delhi, India, \\ 4. Department of Endocrinology, Apex Plus Superspeciality Hospital, Rohtak, India; 5. Department of Endocrinology, Kalpavriksh Superspecialty Center, Dwarka, New \\ Delhi, India; 6. Department of Endocrinology, Manipal Hospitals, Dwarka, New Delhi, India; 7. Department of Endocrinology, Gangaram Bansal Superspecialty Hospital, \\ Sri Ganganagar, India; 8. Department of Endocrinology, Bharti Hospital, Karnal, India
}

DOI: https://doi.org/10.17925/USE.2020.16.2.74

\begin{abstract}
A ims:The knowledge, awareness, and practice with regards to the management of polycystic ovary syndrome(PCOS)among doctorsfrom India is not currently known. This study aimed to determine current clinical practice with regards to managing PCOS. Methods: Local doctors from New Delhi attending an international-led endocrinology conference in New Delhi (the Society for the Promotion of Education in Endocrinology and Diabetes [SPEED] Annual Conference, February 2019) were evaluated regarding their PCOS treatment preferences using a standardized questionnaire, consisting of 30 multiple-choice questions. The questionnaire evaluated doctors' knowledge of clinical symptomatology; biochemical, hormonal, and radiologic evaluation; and pharmacotherapy practices for PCOS. Results: A total of 695 questionnaires were distributed and 538 were collected back, 417 of which had $>80 \%$ of the questions answered and were analyzed (63.31\% of participants were general physicians, $10.31 \%$ were gynecologists, $10.55 \%$ were pediatricians, $6.95 \%$ were endocrinologists, and $8.87 \%$ had other specialties). The mean age of the doctors was $43.07 \pm 13.28$ years. The use of screening for non-classical congenital adrenal hyperplasia by measuring serum 17-OH-progestrone in people living with PCOS is rare in India. Endocrinologists and gynecologists are more likely to use hormone pills to manage PCOS. Both oral contraceptive pills and progestogen-only pills are commonly used in PCOS. Spironolactone is the most commonly used anti-androgen; use of other anti-androgens is low. Metabolic complications of PCOS (dysglycemia and dyslipidemia) are more likely to be screened by endocrinologists and general physicians. Depression in PCOS was more likely to be detected by pediatricians. Conclusion: There is heterogeneity in PCOS treatment practices among doctors of different specialties, warranting better coordination among gynecologists, endocrinologists, pediatricians, and general physicians to homogenize and standardize treatment practices.
\end{abstract}

\section{Keywords}

Polycystic ovary syndrome, clinical practices,

metabolic syndrome, anti-androgens

Disclosures: Deep Dutta, Saptarshi Bhattacharya, Deepak Khandelwal, Sameer Aggarwal, Rajiv Singla, Vineet Surana, Atul Dhingra, and Sanjay Kalra have no financial or non-financial relationships or activities to declare in relation to this article.

Review Process: Double-blind peer review.

Compliance with Ethics: This was a questionnaire-based study among doctors. Informed consent was received from the participants to use their responses in this study. Authorship: The named authors meet the International Committee of Medical Journal Editors (ICMJE) criteria for authorship of this manuscript, take responsibility for the integrity of the work as a whole, and have given final approval for the version to be published.

Access: This article is freely accessible at

touchENDOCRINOLOGY.com @ C Touch Medical Media 2020

Received: February 20, 2020

Accepted: June 24, 2020

Published Online: December 16, 2020

Citation: US Endocrinology. 2020;16(2):74-9

Corresponding Author: Deep Dutta, Department of Endocrinology, Center for Endocrinology, Diabetes, Arthritis \& Rheumatism (CEDAR) Super-specialty Care, Plot 107 and 108, Pocket-A, Sector 12A Dwarka, New Delhi 110078, India. E: deepdutta2000@yahoo.com

Support: No funding was received in the publication of this article.
Polycystic ovary syndrome (PCOS) is a common hormonal, lifestyle, and metabolic disorder, managed by a diverse team of doctors, such as endocrinologists, gynecologists, dermatologists, and internists, depending on the predominant clinical phenotype and the age of presentation of the individual. ${ }^{1}$ Often, there is a lack of coordination between doctors of different specialties, and often the same clinical phenotype may be managed differently by the doctors of different specialties. ${ }^{2}$ This may lead to patient dissatisfaction, confusion, and even depression..$^{2-4}$ Data are not available evaluating the divergence in thought process and treatment practices with regards to doctors from different specialties in India. This study aimed to document the current clinical practices in managing PCOS among doctors of different specialties in New Delhi, focusing on internists, endocrinologists, gynecologists, and pediatricians.

\section{Methods}

Local doctors attending one of the largest international-led endocrinology conferences in New Delhi (Society for the Promotion of Education in Endocrinology and Diabetes [SPEED] Annual Conference, February 2-3, 2019) were evaluated regarding their treatment preferences for PCOS using a standardized questionnaire. At the beginning of the conference, all doctors were given the questionnaire, which included 30 multiple-choice questions, with the scope of marking more than one choice as the preferred answer. A time of 15 minutes was allocated for completing the questionnaire, based on a pilot study performed with 30 doctors before the event. Questions were 
primarily based on evaluating the doctors' preferences and knowledge of clinical symptomatology, biochemical/hormonal/radiologic evaluation, and pharmacotherapy practices

\section{Questionnaire}

Questions 1-6 evaluated participants' knowledge of the clinical symptomatology associated with PCOS. These included questions regarding the age of onset of PCOS in their practice; commonly noticed symptoms and clinical signs; occurrence of obesity, metabolic syndrome, and diabetes; and family history of PCOS. Questions 7-13 evaluated the patterns of radiographical, biochemical, and hormonal evaluation of patients with suspected PCOS. They also evaluated how the doctors confirmed the diagnosis of PCOS. These questions aimed to discover the frequency of use of abdominal ultrasonography, hormone evaluation (gonadotropins, testosterone, dehydroepiandrostenedione sulphate [DHEAS], 17-hydroxy-progestogen [17OHP]), and evaluation of biochemical measures of metabolic syndrome (lipid profile, liver function, serum insulin, glycated hemoglobin [HBA1C], and blood glucose). Serum DHEAS and testosterone are two established biochemical measures of hyperandrogenism in PCOS. Questions 14-19 evaluated treatment and pharmacotherapy practices in PCOS. They evaluated doctors' preferences for the use of therapeutic lifestyle modification, hormone therapy, oral contraceptive pills, anti-androgens (spironolactone, finasteride), and metformin. Questions 20-27 evaluated the doctors' perceptions of the associated complications or disease states with PCOS. These include depression, other endocrinopathies, dysglycemia, infertility, the need for team work and multispecialty therapy, and long-term outcomes. Question 28 was on the clinical specialty of the doctor, question 29 on the gender of the doctor, and question 30 on age of the doctor. The questionnaires were blinded and no other personal details, like name and contact details, were not collected, to avoid any bias while filling of the questionnaires. The detailed questionnaire is included in the supplementary material.

\section{Study participants}

Study participants were a diverse group of practicing doctors ranging from family physicians, internal medicine specialists, gynecologists, pediatricians, and endocrinologists practicing in New Delhi who voluntarily chose to attend the conference. The venue, timing, and the program content of the conference was advertised in the local newspapers, radio, and all major hospitals of the respective cities for 3 months prior to the actual event.

We calculated "weighted percent value" to establish the frequency at which doctors ordered a particular investigation, selecting a particular treatment option, or disease profile with managing PCOS. For questions that had the option to select a particular percentage range $(<10 \%, 10-25 \%$, $25-50 \%, 50-75 \%$, and $>75 \%$ ), a weighted percent value was calculated by multiplying the median value of each of the percent range with number of participants choosing that option, then adding them up for all the five options, and lastly calculating its mean. For $<10 \%$, a median value of 5.0 was considered for calculation; for $10-25 \%$, a median value of 17.5 was considered for calculation; for $25-50 \%$, a median value of 37.5 was considered for calculation; for $50-75 \%$, a median value of 62.5 was considered for calculation; and for $>75 \%$, a median value of 87.5 was used for calculation. This method was used for comparing the management preferences of doctors from different specialties for questions 9-13 and 15-20. The weighted percent value was calculated as the sum of the product of the median of each range with number of doctors selecting that option, and then finding the mean of that for a particular specialty by dividing the sum value with number of doctors of that particular specialty who selected the option. For example, the weighted percent value with regards to frequency of testing for serum 17OHP as a part of PCOS work-up among general physicians was $24.92 \%$. This was achieved by finding the sum of $5 \times 70$ (number of physicians selecting option A) $+17.5 \times 96$ (number of physicians selecting option B) $+37.5 \times 58$ (number of physicians selecting option C) $+62.5 \times 10$ (number of physicians selecting option D) + 87.5 (number of physicians selecting option E) which came to 6,580. The mean of 6,580 for the 264 physicians is $24.92 \%$.

\section{Statistical analysis}

Summary statistics were prepared for responses to each question. Because not every participant answered all questions, the percentage of respondents providing a given answer was calculated individually for each question, using the number of respondents to that question as the denominator. The analysis was performed using the Statistical Package for the Social Sciences (SPSS) (Chicago, LL, USA) version 20.

\section{Results}

A total of 695 questionnaires were distributed to the doctors with their conference kit, at the reception area of the conference. From a total of 538 completed questionnaires that were collected (77.41\%), 417 questionnaires (60.00\%), which fulfilled all criteria, were analyzed. Questionnaires where $<80 \%$ of the questions answered $(n=121)$ were excluded from analysis. The mean age of doctors was $43.07 \pm 13.28$ years; 311 were male, 104 were females, and two doctors chose not to disclose their sex. The specialties of doctors included 264 family physicians/internal medicine specialists (63.31\%), 43 gynecologists (10.31\%), 44 pediatricians (10.55\%), 29 endocrinologists (6.95\%), and 37 doctors of other specialties (8.87\%), which included dermatologists, surgeons, orthopedicians, and psychiatrists, among others.

\section{Polycystic ovary syndrome symptomatology}

The most common age of onset of PCOS, as noted by $62.83 \%$ (262/417) of the doctors, was $18-30$ years. Of the remaining doctors, 136 (32.61\%) and $19(4.55 \%)$ felt that the most common age of presentation was $10-18$ years and 30-40 years, respectively. The clinical symptomatology which made the treating doctors suspect PCOS (in decreasing order) were menstrual irregularity (82.49\%; $n=344)$, abnormal hair growth $(76.26 \%$; $n=318)$, weight gain (74.34\%; $n=310)$, infertility (69.54\%; $n=290)$, acne (59.95\%; $n=250)$, diabetes $(39.33 \% ; 164)$, and hypertension $(15.83 \% ; n=66)$.

Of the doctors who answered the question on PCOS and obesity $(n=369), 171$ doctors (46.34\%) felt that $25-50 \%$ of women with PCOS had associated obesity. Of the remaining doctors, $106(28.72 \%)$ and $56(15.18 \%)$ felt that prevalence of obesity in PCOS was 10-25\% and 50-75\% respectively. Only 36 (9.76\%) doctors felt that prevalence of obesity in PCOS was $<10 \%$. One hundred and four $(24.94 \%), 194(46.52 \%), 84(20.14 \%), 21(5.04 \%)$, and $8(1.92 \%)$ doctors felt that prevalence of diabetes in PCOS was $<10 \%, 10-25 \%, 25-50 \%, 50-75 \%$, and $>75 \%$, respectively. A total of $1.44 \%$ participants $(n=6)$ did not answer this question. A total of 279 doctors (66.91\%) said that they routinely checked waist circumference or calculated waist:hip ratio in people appearing overweight. One hundred and twenty-two (31.20\%), $187(47.82 \%), 68$ (13.85\%), and 14 (3.58\%) doctors felt that people living with PCOS had a positive family history of PCOS in $<10 \%, 10-25 \%, 25-50 \%, 50-75 \%$, and $>75 \%$ in mother/siblings, respectively. A total of $6.23 \%$ participants $(n=26)$ did not answer this question. 
Table 1: Specialty-wise difference in the investigation profile of a person suspected to have polycystic ovary syndrome

\begin{tabular}{|c|c|c|c|c|c|c|}
\hline \multirow[t]{2}{*}{ Investigation } & \multicolumn{5}{|l|}{ Medical specialty } & \multirow[t]{2}{*}{ P-value } \\
\hline & Internist/physician ( $n=264)$ & Gynecologist $(n=43)$ & Pediatrician $(n=44)$ & Endocrinologist $(\mathrm{n}=29)$ & Others $(n=37)$ & \\
\hline USG abdomen and pelvis & 230 (87.12\%) & $34(79.07 \%)$ & 34 (77.27\%) & $24(82.76 \%)$ & $24(64.86 \%)$ & 0.448 \\
\hline Blood glucose & $182(68.94 \%)$ & $31(72.09 \%)$ & $28(63.64 \%)$ & $27(93.10 \%)$ & $17(45.95 \%)$ & 0.079 \\
\hline Liver profile & $90(34.09 \%)$ & $14(32.56 \%)$ & $12(27.27 \%)$ & $14(48.23 \%)$ & $8(21.62 \%)$ & 0.486 \\
\hline
\end{tabular}

P-value calculated using Chi-square test; $p<0.05$ considered statistically significant. USG = ultrasonography.

\section{Polycystic ovary syndrome biochemical-hormonal and radiological evaluation}

A total of 335 doctors answered the question on evaluation of $170 \mathrm{HP}$ in PCOS. Evaluation of 17OHP to screen for non-classical congenital adrenal hyperplasia (NCCAH) was conducted by 142 doctors $(42.38 \%$ ) in $<10 \%$ of their patients with PCOS, and 147 doctors (43.88\%) in 10-25\% of patients with PCOS. Only 26 doctors (7.76\%) and 20 doctors (5.97\%) frequently evaluated $17 \mathrm{OHP}$ in $>75 \%$ and $50-75 \%$ of patients with PCOS, respectively.

A total of 323 doctors answered the question on evaluation of DHEAS in PCOS. DHEAS evaluation was conducted by 112 (34.67\%) and 142 (43.96\%) doctors in $<10 \%$ and $10-25 \%$ of patients with PCOS, respectively. Thirty (9.29\%) and 39 (12.07\%) doctors were frequently evaluating DHEAS in $>75 \%$ and $50-75 \%$ of patients with PCOS, respectively.

A total of 407 doctors answered the question on evaluation of dysglycemia (fasting glucose/HbA1C) in PCOS. Thirty-four (8.35\%), 116 (28.50\%) and $60(14.74 \%)$ doctors conducted dysglycemia screening in <10\%, 10-25\% and $25-50 \%$ of patients with PCOS, respectively. Sixty-six (16.21\%) and 131 (32.19\%) doctors frequently evaluated for dysglycemia in $>75 \%$ and $50-75 \%$ of patients with PCOS, respectively. A total of 403 doctors answered the question on the evaluation of serum insulin levels in patients with PCOS. One hundred and seven (26.55\%), 102 (25.31\%), and 92 (22.82\%) doctors performed serum insulin assessment in <10\%, 10-25\%, and $25-50 \%$ of patients with PCOS, respectively. Forty-eight (11.91\%) and 54 (13.39\%) doctors frequently evaluated serum insulin levels in $>75 \%$ and $50-75 \%$ of patients with PCOS, respectively. A total of 399 doctors answered the question on evaluation of lipid profile in PCOS. Sixty-four (16.04\%), 100 (25.06\%), and 95 (23.08\%) doctors assessed serum lipid profile in $<10 \%$, 10-25\%, and $25-50 \%$ of patients with PCOS, respectively, while 54 (13.53\%) and 86 (21.55\%) doctors frequently evaluated for lipid profile in $>75 \%$ and $50-75 \%$ of patients with PCOS, respectively.

Overall if we look at the preference for different investigations in the diagnostic work-up of patients with PCOS, the most popular investigation was abdominal ultrasound ( $n=360)$, followed by hormone analysis $(n=314)$, blood glucose testing ( $n=285)$, dyslipidemia $(n=188)$, and liver function test $(n=138)$ (see Table 1).

\section{Polycystic ovary syndrome pharmacotherapy}

A total of 333 doctors answered the question on the use of oral contraceptive pills (OCPS) for managing PCOS. Seventy (21.02\%), 109 (32.73\%), and 104 (31.23\%) doctors reported using OCPs to treat PCOS in <10\%, 10-25\%, and $25-50 \%$ of patients, respectively. Forty-eight (14.41\%) and 28 (8.41\%) doctors frequently used oral contraceptives in $>75 \%$ and $50-75 \%$ of patients, respectively. A total of 413 doctors answered the question on the use of short-term progestogens for managing PCOS. Eighty-three (20.09\%), $118(28.57 \%)$, and 64 (15.49\%) doctors used short-term progestogen in $<10 \%, 10-25 \%$, and $25-50 \%$ of patients with PCOS, respectively. Eighty-four (20.33\%) and 64 (15.49\%) doctors frequently used short-term progestogen in $>75 \%$ and $50-75 \%$ of patients, respectively.

A total of 349 doctors answered the question on the use of spironolactone as an anti-androgen for managing PCOS. The treatment of PCOS with spironolactone was reported by 136 (38.96\%), 120 (34.38\%), and 60 (17.19\%) doctors in $<10 \%, 10-25 \%$, and $25-50 \%$ of patients, respectively. Twenty (5.73\%) and thirteen (3.72\%) doctors reported frequently using spironolactone in $>75 \%$ and $50-75 \%$ of patients, respectively. A total of 333 doctors answered the question on use of finasteride as an anti-androgen for managing PCOS. Use of finasteride as an anti-androgen was reported by 186 (55.85\%), 87 (26.12\%), and 50 (15.01\%) doctors in <10\%, 10-25\%, and $25-50 \%$ of patients with PCOS, respectively. Two and 8 doctors reported the use of finasteride in $50-75 \%$ and $>75 \%$ of patients with PCOS, respectively. A total of 331 doctors answered the question on use of metformin for managing PCOS. Twenty (6.04\%), 58 (17.52\%), and 113 (34.14\%) doctors reported using metformin to treat PCOS in $<10 \%, 10-25 \%$, and $25-50 \%$ of patients respectively. Sixty-six (19.93\%) and 74 (22.35\%) doctors frequently used metformin in $>75 \%$ and $50-75 \%$ of patients, respectively.

\section{Polycystic ovary syndrome-associated disorders and outcomes}

A total of 327 doctors answered the question on the burden of depression in women living with PCOS. Seventy (21.40\%), 117 (35.78\%), and 94 (28.74\%) doctors felt depression occurred in $<10 \%, 10-25 \%$, and $25-50 \%$ of patients with PCOS respectively. Whereas 32 (9.78\%), and 14 (4.28\%) doctors felt that depression was common in patients with PCOS, occurring in $>75 \%$ and $50-75 \%$ of patients, respectively.

\section{Polycystic ovary syndrome treatment practices- variation among different specialties}

170 HP testing was most commonly ordered by endocrinologists, followed by the general physicians, and least by the pediatricians, which was statistically significant. There was no significant difference in DHEAS and insulin testing among doctors of different specialties. Screening for dysglycemia and dyslipidemia was most commonly performed by endocrinologists, followed by general physicians, and most infrequently by doctors of other specialties and it was statistically different. The use of estrogen + progestogen combination pills for the treatment of PCOS was statistically different among 
Table 2: Weighted percent value for frequency of ordering a particular investigation, selecting a particular treatment option, or disease profile with managing polycystic ovary syndrome

\begin{tabular}{|c|c|c|c|c|c|c|}
\hline \multirow[t]{2}{*}{ Diagnostic preferences } & \multicolumn{5}{|l|}{ Medical specialty } & \multirow[t]{2}{*}{ P-value } \\
\hline & $\begin{array}{l}\text { Internists/general } \\
\text { physicians } \\
(\mathrm{n}=264)\end{array}$ & $\begin{array}{l}\text { Gynecologists } \\
(n=43)\end{array}$ & $\begin{array}{l}\text { Pediatricians } \\
(n=44)\end{array}$ & $\begin{array}{l}\text { Endocrinologists } \\
(n=29)\end{array}$ & $\begin{array}{l}\text { Others } \\
(n=37)\end{array}$ & \\
\hline 170H-progestrone testing (Q9) & $24.92 \%$ & $19.24 \%$ & $12.95 \%$ & $38.36 \%$ & $14.66 \%$ & $<0.001$ \\
\hline DHEAS testing (Q10) & $26.98 \%$ & $28.55 \%$ & $22.5 \%$ & $28.53 \%$ & $15.88 \%$ & 0.166 \\
\hline FBG/HbA1C testing (Q11) & $45.02 \%$ & $38.43 \%$ & $44.20 \%$ & $69.57 \%$ & $26.68 \%$ & $<0.001$ \\
\hline Serum insulin (Q12) & $33.10 \%$ & $23.26 \%$ & $28.64 \%$ & $33.10 \%$ & $21.22 \%$ & 0.183 \\
\hline Lipid profile (Q13) & $43.01 \%$ & $30.58 \%$ & $38.52 \%$ & $44.39 \%$ & $19.73 \%$ & 0.002 \\
\hline \multicolumn{7}{|l|}{ Treatment preferences } \\
\hline E+P pills (Q15) & $27.23 \%$ & $31.97 \%$ & $25.45 \%$ & $53.02 \%$ & $11.08 \%$ & $<0.001$ \\
\hline Spironolactone as antiandrogen (Q16) & $18.33 \%$ & $12.67 \%$ & $14.55 \%$ & $43.02 \%$ & $3.92 \%$ & $<0.001$ \\
\hline Finasteride as antiandrogen (Q17) & $12.82 \%$ & $15.46 \%$ & $12.5 \%$ & $11.64 \%$ & $5.81 \%$ & 0.343 \\
\hline Short-term progestogens (Q18) & $20.85 \%$ & $25.12 \%$ & $20.34 \%$ & $21.37 \%$ & $3.51 \%$ & 0.002 \\
\hline Metformin (Q19) & $42.08 \%$ & $38.13 \%$ & $35.22 \%$ & $44.74 \%$ & $9.59 \%$ & $<0.001$ \\
\hline \multicolumn{7}{|l|}{ Patient factors } \\
\hline Depression (Q20) & $23.63 \%$ & $15.52 \%$ & $30.80 \%$ & $25.77 \%$ & $3.72 \%$ & $<0.001$ \\
\hline
\end{tabular}

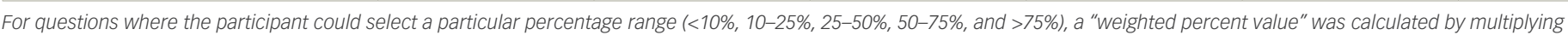

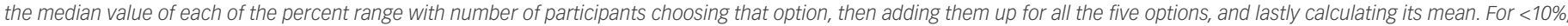
a median value of 5 was considered for the calculation; for 10-25\%, a median value of 17.5 was considered for calculation; for $25-50 \%$, a median value of 37.5 was considered for calculation; for 50-75\%, a median value of 62.5 was considered for calculation; and for $>75 \%$, a median value of 87.5 was used for calculation. This method was used for comparing


range with number of doctors selecting that option and then finding the mean of that for a particular specialty by dividing the sum value with number of doctors of that particular specialty who filed the option.

DHEAS = dehydroepiandrostenedione sulphate; $E+P=$ estrogen and progestogen; FBG = fasting blood glucose; HbA1C = glycated hemoglobin; $Q=$ question .

groups, with highest being in endocrinologists. Use of spironolactone as an anti-androgen was also statistically different among groups, with the highest use by endocrinologists, followed by gynecologists. Finasteride use as an anti-androgen was low and similar between the groups. The use of short-term progestogen was significantly different among groups, with the highest level among gynecologists for women with oligomenorrhea. The use of metformin was highest among endocrinologists, followed by general physicians, and was lowest in doctors of other specialties. Detection of depression in girls living with PCOS was highest by pediatricians. Weighted percent value can be seen in Table 2 .

\section{Discussion}

Our study reiterated that PCOS is predominantly a disease of young women; peak age of diagnosis was reported to be 18-30 years in our study, noted by $63 \%$ of the doctors. Menstrual irregularity, hirsutism, weight gain, infertility, and acne were the top five signs and symptoms noted by doctors which lead to the diagnosis of PCOS. Obesity is being increasingly documented in PCOS. ${ }^{1,4}$ This is supported by the observation that the majority of the doctors in this study felt their patients with PCOS had associated obesity. Positive family history of PCOS was not commonly noted in patients with PCOS.

In a population-based study screening 2,400 women from Haryana, India, the prevalence of PCOS was observed to be $4.21 \%$, with $71 \%$ patients being from urban populations. ${ }^{5}$ Biochemical hyperandrogenism was the most common phenotype among people living with PCOS (64\%), followed by menstrual irregularities (52\%), ultrasound appearances of polycystic ovaries (35\%), and clinical hirsutism (30\%). ${ }^{5}$ All the three features of polycystic ovaries, along with clinical hirsutism and biochemical hyperandrogenism, were present in only $19 \%$ patients. ${ }^{5}$ In a multi-country study evaluating racial and ethnic differences in the prevalence of metabolic syndrome in people with PCOS, women from India had the highest mean Ferriman-Gallwey score for clinical hyperandrogenism, age-adjusted odds ratio for metabolic syndrome was highest in African-American women. ${ }^{6}$

Screening for NCCAH using 17OHP and hyperandrogenism was low among doctors in this study. Only $6.24 \%$ doctors routinely screened for serum $170 \mathrm{HP}$ to rule out NCCAH in their practice. Endocrinologists were most likely to screen for NCCAH among doctors of different specialties. Uliassi et al. also noted that endocrinologists were more likely to screen for serum morning cortisol and congenital adrenal hyperplasia compared to general physicians and the gynecologists. ' Hence, there is a need to increase awareness among doctors managing patients with PCOS with regards to NCCAH. DHEAS testing was quite popular with regards to screening for biochemical evidence of hyperandrogenism. DHEAS is typically a measure of the degree of adrenal hyperandrogenism, whereas serum testosterone is a measure of ovary-associated hyperandrogenism. ${ }^{8}$

Screening for dysglycemia (fasting glucose and/or $\mathrm{HbA} 1 \mathrm{c}$ ) was much more frequent than screening for biochemical evidence of hyperandrogenism. Endocrinologists and general physicians were most likely to screen for dysglycemia and dyslipidemia among doctors of different specialties. Uliassi et al. also noted that endocrinologists and generalists/physicians were more likely to order lipid profile evaluations in women with PCOS. In their study of prescription records, $60 \%$ of endocrinologists and generalists/physicians ordered lipid profile tests for their patients with PCOS, compared with none 
by the gynecologists. ${ }^{7}$ This is especially relevant from an Indian perspective, in view of the high prevalence of both prediabetes (11-14\%) and diabetes (9-11\%) among Indians. ${ }^{9}$ Indians develop type 2 diabetes nearly 2 decades earlier than the western world, with peak incidence around 30 years of age, when PCOS prevalence is also fairly high in women. ${ }^{10}$ Nearly one-quarter of the doctors (24.46\%) frequently evaluated for serum insulin in women living with PCOS. Raised serum insulin may be a marker of insulin resistance. Additionally, serum insulin is used to calculate surrogate measures of insulin resistance, such as the Homeostasis Model Assessment of Insulin Resistance (HOMA-IR) index and the Quantitative Insulin Sensitivity Check Index (QUICKI), among others. ${ }^{11}$ However, their routine use in clinical practice is discouraged, and we rarely get any new information to help change clinical practice. ${ }^{12}$ The HOMA-IR index and QUICKI are more relevant for research purposes and for epidemiologic studies; absolute values have limited utility. ${ }^{12}$

Clinical measures of insulin resistance (acanthosis, skin tags, central obesity) are good markers to modulate treatment plans and considerations, such as whether or not to use metformin. Metformin is used in patients with PCOS for reduction in associated insulin resistance, weight loss, and also plays a role in improving fertility. ${ }^{13}$ Dyslipidemia is not uncommon in PCOS as a part of metabolic syndrome. Increased insulin resistance in PCOS is responsible for atherogenic dyslipidemia, characterized by raised triglycerides, mildly raised low-density lipoprotein cholesterol with low high-density lipoprotein cholesterol. ${ }^{3}$ Lipid profile testing as a screening method for dyslipidemia was not commonly conducted in women with PCOS by doctors in our study, with only $12.95 \%$ of doctors routinely screening for dyslipidemia. To summarize, abdominal ultrasound, followed by hormone analysis, blood glucose testing, screening for dyslipidemia, and liver function test were the most popular investigations in women suspected to have PCOS. In a study from USA, reproductive endocrinologists were more aware of the diagnostic criteria for PCOS, compared with gynecologists. ${ }^{14}$ The Rotterdam Criteria were the most popular and commonly used diagnostic criteria. Gynecologists were less likely to be aware of depression, anxiety disorders, and reduced quality of life associated with PCOS, compared with reproductive endocrinologists. ${ }^{14}$

Estrogen plus progestogen-containing oral contraceptive pills are used in patients with PCOS to correct persistent hyperestrogenism, reduce hyperandrogenism, and regularize menstrual cycles. ${ }^{15}$ They are also believed to have a beneficial impact on future fertility in women with irregular menstrual cycles. ${ }^{16}$ Nearly one in five doctors evaluated frequent used oral contraceptives for managing PCOS. Short-term progestogen (medroxyprogesterone acetate/norethisterone among others) are often used in hyperestrogenism associated with PCOS to cause breakthrough menstrual flow. Short-term progestogens were more popular than oral contraceptive in managing PCOS, with one in three doctors evaluated frequently using them in clinical practice. Oral contraceptive pills are associated with the problems of fluid retention, weight gain, worsening of migraine, and may sometimes be associated with dyslipidemia and transaminitis, all of which are more relevant in overweight to obese patients with PCOS. Advantages of short-term progestogens over oral contraceptive pills include the need for shorter course of therapy of 3-7 days, and they are not associated with weight gain or metabolic abnormalities.

Spironolactone was the most popular anti-androgen used to manage PCOS in our study. Use of finasteride as an anti-androgen was low across doctors from all specialties. This is in accordance with treatment practices in USA. ${ }^{17}$ In the USA, spironolactone is the most popular anti-androgen, followed by finasteride, flutamide, and dutasteride. ${ }^{17}$ In the current study, endocrinologists and gynecologists were the two groups of doctors who are most likely to use anti-androgens for managing PCOS. Metformin use was common among doctors managing PCOS, with more than $50 \%$ of doctors in this study frequently using metformin in their practice. Metformin has been found to be beneficial both in lean and obese patients for reducing the symptoms of PCOS, in conjunction with oral contraceptive pills and anti-androgens. ${ }^{17}$ Metformin for PCOS was more commonly prescribed by endocrinologists, compared with other specialties in the study by Uliassi et al.?

The chronic nature of PCOS, associated with altered physical appearance (excess body hair, acne, obesity, metabolic syndrome), menstrual irregularities, and infertility may lead to depression is some patients. ${ }^{4}$ In our study, depression was commonly noted by one in three doctors in their patients living with PCOS. Depression was most likely to be picked up by pediatricians in our study, highlighting that it may be a significant problem in young girls with PCOS in their teenage years. In a meta-analysis involving 12 studies where depression was primarily assessed (910 women with PCOS and 1,347 controls) and six studies where anxiety was primarily assessed (208 women with PCOS and 169 controls), both the mental health manifestations were found to be associated with PCOS. ${ }^{18}$ Women with higher body mass index had worse depression and anxiety scores. ${ }^{18}$ In a study from the USA evaluating treatment practices in adolescent patients with PCOS, gynecologists were more likely to order pelvic ultrasonography than pediatricians and endocrinologists, whereas metformin use was highest by endocrinologists, followed by gynecologists and pediatricians, highlighting that heterogeneity in PCOS treatment practices is a global phenomenon. ${ }^{19}$ It must be highlighted that the low participation of endocrinologists in the study cohort is reflective of the small number of endocrinologists in clinical practice in India, compared with doctors from other specialties. ${ }^{20}$

To summarize, this is the first study, to our knowledge, to evaluate the treatment practices in women living with PCOS by doctors of different specialties in India. Screening for NCCAH in people living with PCOS is rare in India. Endocrinologists and gynecologists are more likely to use hormone pills to manage PCOS. Metabolic complications of PCOS are more likely to be screened by endocrinologists and general physicians. As depression was detected more frequently by pediatricians, this indicates that depression is more likely to be faced by young girls with PCOS. There is an urgent need for more interaction and collaboration among gynecologists, endocrinologists, pediatricians, and general physicians to homogenize and standardize the treatment practices in India. $\square$
1. Wolf WM, Wattick RA, Kinkade ON, Olfert MD. The current description and future need for multidisciplinary PCOS clinics. J Clin Med. 2018;7:395.

2. Gibson-Helm M, Teede H, Dunaif A, Dokras A. Delayed diagnosis and a lack of information associated with dissatisfaction in women with polycystic ovary syndrome. I Clin Endocrinol Metab. 2017;102:604-12.
3. Sawant S, Bhide P. Fertility treatment options for women with polycystic ovary syndrome. Clin Med Insights Reprod Health. 2019;13:1179558119890867.

4. Alur-Gupta S, Chemerinski A, Liu C, et al. Body-image distress is increased in women with polycystic ovary syndrome and mediates depression and anxiety. Fertil Steril. 2019:112:930-8.e1.

5. Deswal R, Nanda S, Ghalaut VS, et al. Cross-sectional study of the prevalence of polycystic ovary syndrome in rural and urban populations. Int J Gynaecol Obstet. 2019;146:370-9.

6. Chan JL, Kar S, Vanky E, et al. Racial and ethnic differences in the prevalence of metabolic syndrome and its components of metabolic syndrome in women with polycystic ovary syndrome: a regional cross-sectional study. Am J Obstet Gynecol. 2017;217:189.e1-8. 
7. Uliassi N, Sullivan S, Damle L, Gomez-Lobo V. Trends, in diagnosis and treatment of polycystic ovarian syndrome by specialty. J Reprod Med. 2016;61:441-6.

8. Luque-Ramírez M, Escobar-Morreale HF. Adrenal hyperandrogenism and polycystic ovary syndrome. Curr Pharm Des. 2016;22:5588-602.

9. Dutta D, Mukhopadhyay S. Intervening at prediabetes stage is critical to controlling the diabetes epidemic among Asian Indians. Indian J Med Res. 2016;143:401-4.

10. Dutta D, Ghosh S. Young-onset diabetes: an Indian perspective Indian J Med Res. 2019;149:441-2.

11. Dutta D, Maisnam I, Shrivastava A, et al. Serum vitamin-D predicts insulin resistance in individuals with prediabetes. Indian Med Res. 2013;138:853-60.

12. Dutta D, Maisnam I, Shrivastava A, et al. Authors' response. Indian J Med Res. 2014;139:959-60.

13. Jungheim $E S$, Odibo $A O$. Fertility treatment in women with polycystic ovary syndrome: a decision analysis of different ora ovulation induction agents. Fertil Steril. 2010;94:2659-64.

14. Dokras A, Saini S, Gibson-Helm M, et al. Gaps in knowledge among physicians regarding diagnostic criteria and management of polycystic ovary syndrome. Fertil Steril. 2017:107:1380-6.e1.

15. Liu Q, Xie YJ, Qu LH, et al. Dyslipidemia involvement in the development of polycystic ovary syndrome. Taiwan J Obstet Gynecol. 2019;58:447-53

16. Amiri M, Kabir A, Nahidi F, et al. Effects of combined oral contraceptives on the clinical and biochemical parameters of hyperandrogenism in patients with polycystic ovary syndrome: a systematic review and meta-analysis. Eur J Contracept Reprod Health Care. 2018;23:64-77.

17. Goodman NF, Cobin RH, Futterweit W, et al, American Association of Clinical Endocrinologists (AACE), American College of Endocrinology (ACE), Androgen Excess and PCOS Society (AES).
American Association of Clinical Endocrinologists, American College of Endocrinology, and Androgen Excess and PCOS Society disease state clinical review: Guide to the best practices in the evaluation and treatment of polycystic ovary

18. Barry JA, Kuczmierczyk AR, Hardiman PJ. Anxiety and depression in polycystic ovary syndrome: a systematic review and in polycystic ovary syndrome: a systematic reverw

19. Powers SE, Uliassi NW, Sullivan SD, et al. Trends in standard workup performed by pediatric subspecialists for the diagnosis of adolescent polycystic ovary syndrome. J Pediatr Adolesc Gynecol. 2015;28:43-6

20. Khandelwal $D$, Dutta $D$, Singla R, et al. Perceptions about training during endocrinology residency programs in India over the years: a cross-sectional study (PEER India Study). Indian J Endocrinol Metab. 2017:21:271-6. 